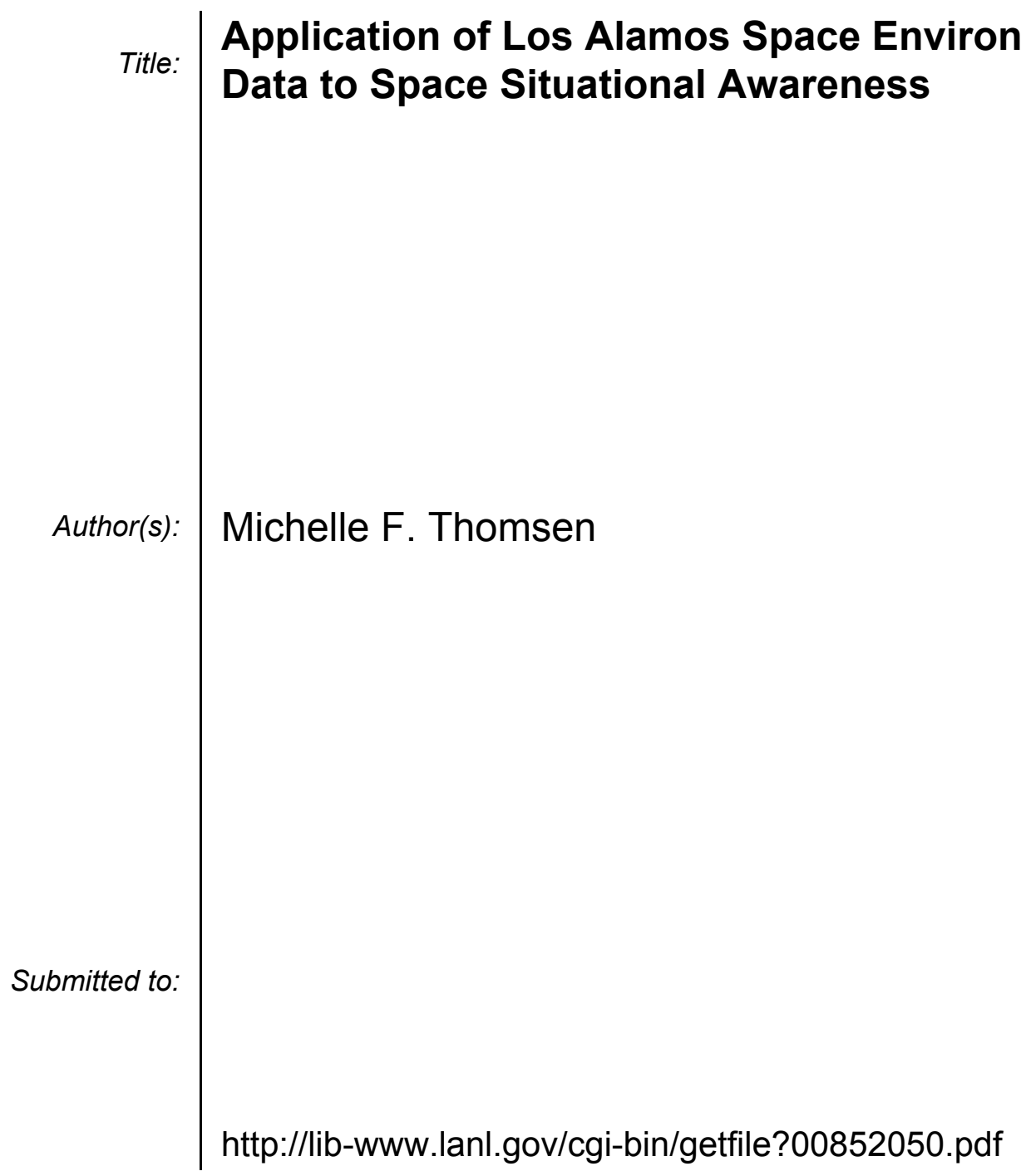

Los Alamos National Laboratory, an affirmative action/equal opportunity employer, is operated by the University of California for the U.S. Department of Energy under contract W-7405-ENG-36. By acceptance of this article, the publisher recognizes that the U.S. Government retains a nonexclusive, royaltyfree license to publish or reproduce the published form of this contribution, or to allow others to do so, for U.S. Government purposes. Los Alamos National Laboratory requests that the publisher identify this article as work performed under the auspices of the U.S. Department of Energy. Los Alamos National Laboratory strongly supports academic freedom and a researcher's right to publish; as an institution, however, the Laboratory does not endorse the viewpoint of a publication or guarantee its technical correctness. 


\title{
Application of Los Alamos Space Environment Data to Space Situational Awareness
}

\author{
Michelle F. Thomsen, mthomsen@lanl.gov
}

\begin{abstract}
The magnetospheric environment in which military satellites must operate is very dynamic and exhibits spatial and temporal variations that have been termed "space weather." Space weather conditions can have significant adverse effects on orbital assets. Los Alamos has for many years been monitoring the space environment at geosynchronous orbit and has accumulated a large database of magnetospheric charged particle measurements. The objective of this project was to exploit these existing LANL databases to address important problems that affect space situational awareness for military satellites. The tasks conducted were directed toward the proof of principle of the utility of space environment measurements for predicting and detecting potentially harmful space weather conditions and/or hostile actions aimed at military space assets. By analyzing this very large database, we demonstrated a clear and previously unknown solar-cycle dependence to the typical fluxes and plasma-sheet densities encountered at geosynchronous orbit, with maximum densities observed during years of maximum solar activity. Moreover, we showed that the plasma-sheet densities are quite different during geomagnetic storms compared to normal conditions. We also determined the dependence of strong satellite surface charging on ambient magnetospheric properties and solar wind driving conditions. Finally, we began a collaboration with colleagues at the Aerospace Corporation to include Los Alamos data in their analyses supporting the Defense Counter-Space TestBed initiative.
\end{abstract}

\section{Background and Research Objectives}

The magnetospheric environment in which military satellites must operate is far from static and benign. Driven by variations in the plasma and magnetic field of the solar wind, the earth's magnetosphere is very dynamic and exhibits spatial and temporal variations that have been termed "space weather." Space weather conditions can have significant adverse effects on orbital assets. For example, hot clouds of energized plasma injected into the near-earth region during so-called magnetospheric substorms can cause severe surface charging of satellites, leading to differential charging, consequent electrical discharges, and solar panel degradation. Additionally, subsequent to major magnetic storms (which also produce disruption of ground assets, such as power distribution systems), the outer electron radiation belts can be greatly enhanced, leading to intense fluxes of relativistic electrons that can disable or even kill satellites through deep dielectric charging. 
In addition to the hazards associated with naturally occurring space weather events, military satellites could conceivably be vulnerable to the attempts of hostile parties to probe or even disable them. Such attempts would potentially produce signatures in various state-of-health or event-monitoring instruments on the target satellite, signatures that must be distinguished from the response to natural environmental variations if they are to provide useful intelligence about the attack or interrogation.

Military satellite operators are increasingly aware of and concerned about space weather hazards and about the potential for hostile actions against their assets. They need improved understanding of the nature of the natural space environment and a clear statistical picture of the likelihood of encountering adverse conditions. They also need a way to discriminate between the effects of the natural environment and the effects of a hostile advance.

For over a decade, Los Alamos has flown a very capable suite of space environmental monitors as part of a DOE payload on a number of DoD satellites in geosynchronous orbit. Geosynchronous orbit, where the orbital period is exactly one day, so that a satellite in that orbit stays continuously over the same point on the surface of the earth, is heavily populated with commercial and military assets. Moreover, the magnetospheric environment there is particularly dynamic, and geosynchronous conditions are something of a bellwether for the rest of the magnetosphere. The Los Alamos instruments cover the full range of magnetospherically relevant charged-particle energies, from cold plasmas of ionospheric origin to extremely energetic radiation belt particles. With multiple satellites, we have excellent spatial and temporal coverage of geosynchronous conditions over the full range of geomagnetic activity, for an entire solar cycle.

The objective of this project was to bring to bear these existing LANL databases on important problems that affect space situational awareness for military satellites. This includes knowledge of the natural space environment, its variability, and its effects on military assets in space, as well as detection of possible attack or interrogation of those assets.

\section{Importance to LANL's Science and Technology Base and National R\&D Needs}

The project was specifically relevant to the Laboratory's strategic goal of developing new partnerships with the Department of Defense to address important national and global issues. 
The tasks conducted were directed toward the proof of principle of the utility of space environment measurements for predicting and detecting potentially harmful space weather conditions and/or hostile actions aimed at military space assets.

\section{Scientific Approach and Accomplishments}

The project involved three specific tasks, all utilizing the extensive, multi-year, multi-satellite database of geosynchronous space environment observations obtained by Los Alamos instruments on DoD satellites. Those tasks and our progress on each of them during the funded interval are as follows:

Task 1: Using measurements of plasma and energetic particles over more than an entire solar cycle, perform a statistical characterization of the geosynchronous space environment.

Progress: We have examined the fluxes and moments of the geosynchronous plasma electron and ion populations as a function of local time (LT) and magnetospheric activity (Kp) for each year of an entire solar cycle (11 years). For each year, the LT/Kp-dependence of the fluxes is wellorganized by a fairly simple transport model described in detail in our earlier publication [Korth et al., 1999]. However, our extension of the analysis to the full solar cycle demonstrates a very clear and previously unknown solar-cycle dependence to the typical fluxes and plasma-sheet densities encountered at geosynchronous orbit. This variation is illustrated in Figure 1, which shows the color-coded average electron densities observed by several geosynchronous satellites for each year of the solar cycle. Each panel shows the density sorted according to local time (orbital phase relative to local midnight) on the horizontal axis and magnetospheric activity index (Kp) on the vertical axis. The densities are clearly lower during the years of solar activity minimum (1995-7) compared to solar activity maximum (1990-2 and 1999-2000), at essentially all Kp levels. Comparing these densities with the corresponding ion densities, computed under the assumption that all of the ions are protons, we have found strong evidence that the density variation seen in Figure 1 is due primarily to the addition of ionospheric oxygen to the magnetospheric population during solar maximum conditions. These results are currently being prepared for publication. We have similarly explored the statistical behavior of several other relevant quantities, including the magnetospheric-storm-time behavior. We find that during geomagnetic storms, the plasma-sheet densities on the dawn side of the magnetosphere are significantly enhanced, but the temperatures are appreciably lower than normal times, suggesting a more direct access of shocked solar wind plasma into the near-earth region under these condition. These results are also being prepared for publication. 
Task 2: Use the same database to analyze statistically the conditions that produce satellite surface charging.

Progress: Figure 2 summarizes one aspect of the chain of relationships we have established between our spacecraft surface potential and the environment at geosynchronous orbit and in the solar wind. Figure 2 a shows that there is a threshold hot-electron flux needed to initiate strong surface charging: no charging above 20 volts is observed unless the electron flux exceeds $\sim 900 \mathrm{eV}$ $\mathrm{cm}^{-3}$. For this class of events (i.e., for fluxes exceeding that threshold), Figure $2 \mathrm{~b}$ shows that there is a strong correlation between the size of the surface potential and the value of the electron temperature. Further, Figure $2 \mathrm{~b}$ shows two separated populations of points, corresponding to eclipse intervals (upper population) and non-eclipse intervals (lower-population). Without photoelectrons available to partially neutralize the hot-electron bombardment, the surface potential in eclipse is typically an order of magnitude higher than for the same environment in sunlight. Our earlier work [Borovsky et al., 1998] demonstrated a clear linear relationship between the night-side electron temperature at geosynchronous orbit and the solar wind flow velocity. On the basis of that finding, plus Figures $2 \mathrm{a}$ and $2 \mathrm{~b}$, we expect that the surface charging in the geosynchronous environment should be related to the solar wind velocity, and this is demonstrated in Figure $2 \mathrm{c}$. Figure $2 \mathrm{c}$ shows the cross-correlation coefficient between the spacecraft potential measured at geosynchronous orbit near and after local midnight and the time-lagged solar wind speed. Both curves show definite but broad peaks near 0 hours time lag, demonstrating the expected relationship. The relatively broad peaks are attributable to the long autocorrelation times for the solar wind speed itself. A report describing these relationships is in preparation.

Task 3: In collaboration with colleagues at Aerospace Corporation, contribute to the Defense Counter-Space Test-Bed initiative, in which the full record of state-of-health and event data from a satellite with environmental monitors is examined for anomalous signatures.

Progress: Because of program timing issues at Aerospace, our colleagues were not in position to use our data in their study until quite late in the fiscal year. We have nonetheless provided them with statistical information regarding the extremes in flux levels that are observed by our instruments in geosynchronous orbit, and we will be expanding this collaboration in the near future. 
The studies briefly summarized here demonstrate the proof of principle of the utility of space environment measurements for predicting and detecting potentially harmful space weather conditions and/or hostile actions aimed at military space assets. Related efforts will be proposed for NSF and NASA support within the National Space Weather Program.

\section{Publications}

1. Korth, H., and Thomsen, M. F., "Solar-cycle dependence of the near-earth plasma sheet density," J. Geophys. Res. , in preparation (2001).

2. Thomsen, M. F., Korth, H., Lynch, S. D., and Delapp, D., "Storm-time behavior of the geosynchronous plasma-sheet density," J. Geophys. Res., in preparation (2001).

\section{References}

Borovsky, J. E., M. F. Thomsen, and R. C. Elphic, The driving of the plasma sheet by the solar wind, J. Geophys. Res., 103, 17617, 1998.

Korth, H., M. F. Thomsen, J. E. Borovsky, and D. J. McComas, Plasma sheet access to geosynchronous orbit, J. Geophys. Res., 104, 25047, 1999.

\section{Figure Captions}

1 Average plasma-sheet electron density at geosynchronous orbit for each year of the 11-year solar cycle, sorted by orbital phase (local time) and magnetospheric activity (Kp). The densities are clearly lower during the years of solar activity minimum (1995-7) than during the years of solar maximum (1990-2 and 1999-2000).

2 Chain of relationships between spacecraft surface potential and space environment (locally and within the solar wind): a) strong charging requires a threshold hot-electron flux; 2) when the threshold flux is exceeded, the charging level is related to the ambient hot-electron temperature, with a higher potential produced during eclipse (upper population) than in sunlight (lower population); c) finally, because the plasma-sheet electron temperature is related to the solar wind speed [Borovsky et al., 1998], there is also a correlation between the surface potential of satellites in geosynchronous orbit and the solar wind speed. 

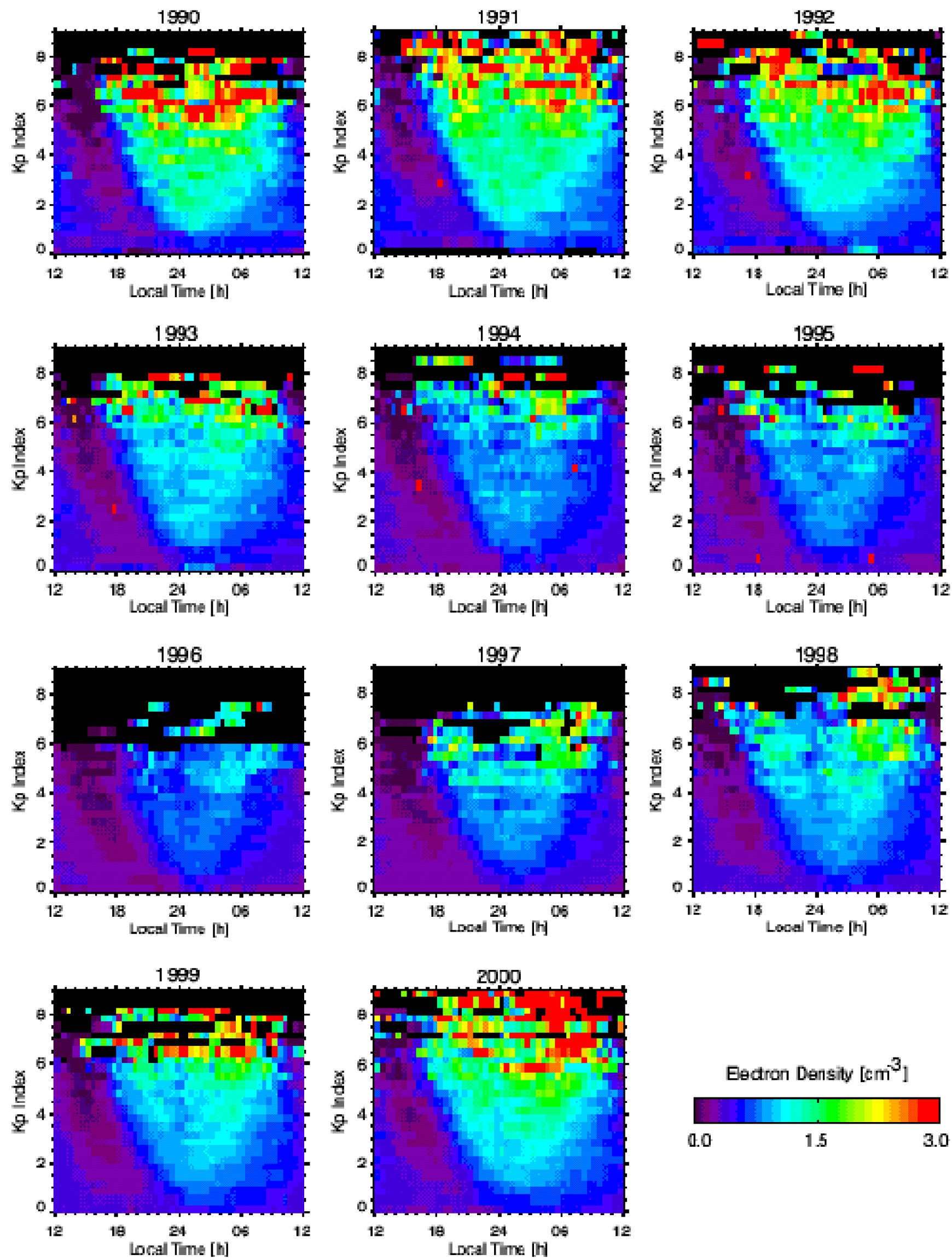

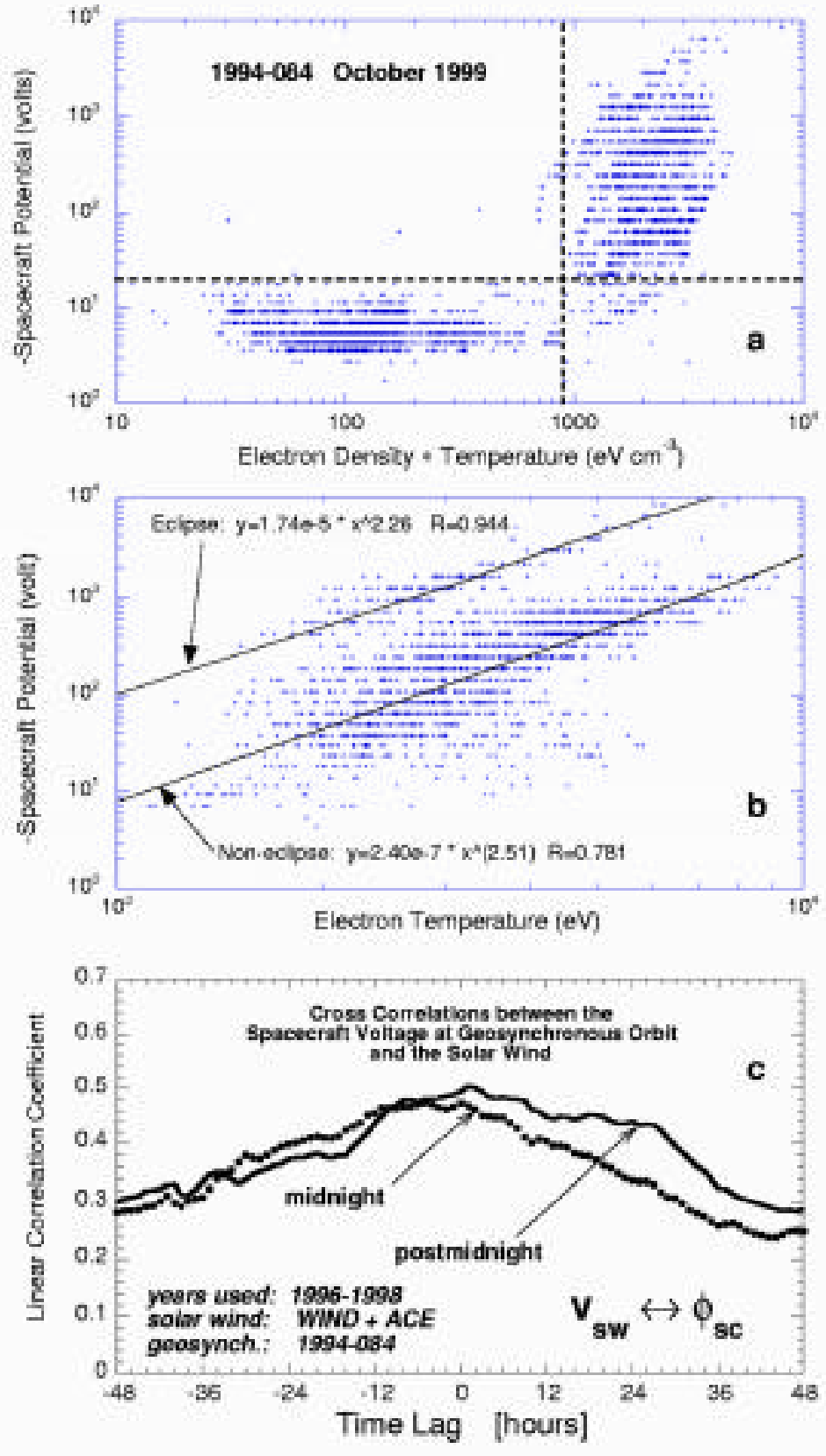